\title{
Observations of parasitism and aggression by female Enoggera nassaui, an egg parasitoid of the eucalypt pest, Paropsis charybdis
}

Sarah Mansfield ${ }^{1,2,3}$ (1)

\begin{abstract}
Background: Female parasitoids that display aggressive behaviour towards conspecifics in defence of host resources must make a trade-off between time spent on aggression and time spent on parasitism. Such a trade-off not only affects the fitness of individual females but also may affect the overall rate of parasitism in the host population. Enoggera nassaui is an egg parasitoid of the eucalypt pest, Paropsis charybdis, with females that defend batches of host eggs from conspecific females by chasing, pushing and sometimes biting their opponent.

Methods: The behaviour of female E. nassaui was observed when alone (solo) and in pairs (owner-intruder) in response to a single batch of $P$. charybdis eggs. The first female in a pair to contact the host eggs was designated the owner and the second female the intruder. Both parasitism-related behaviour for solo and paired females and aggressive interactions between paired females were described from direct observation. Parasitism rates for solo and paired females were also measured.

Results: Solo females had a consistent pattern of parasitism-related behaviour, spending $80 \%$ of their time in contact with the host eggs. Paired females showed a similar pattern of parasitism-related behaviour when not interrupted by their competitor. Owners spent $6 \%$ and intruders spent $17 \%$ of their time on aggressive interactions. Intruders completed parasitism faster than solo females because intruders reduced the duration of specific parasitism-related behaviours. Yet from the host's perspective, parasitism by solo females (69\%) was similar to that achieved by both owners and intruders together (66\%).

Conclusions: By completing parasitism faster in the presence of a competitor, intruder females compensated to some extent for time spent on aggressive interactions. If $E$. nassaui has a similar response to intraspecific competitors under natural conditions, then aggression between conspecific females is unlikely to harm pest suppression by these parasitoids.
\end{abstract}

Keywords: Biological control, Chrysomelidae, Eucalypts, Intraspecific competition, Pteromalidae

Correspondence: sarah.mansfield@agresearch.co.nz

${ }^{1}$ Scion (New Zealand Forest Research Institute Ltd.), Rotorua 3046, New

Zealand

${ }^{2}$ Present Address: AgResearch, Lincoln, Private Bag 4749, Christchurch 8140,

New Zealand

Full list of author information is available at the end of the article 


\section{Background}

Interference competition occurs between female parasitoids that defend host patches aggressively against conspecifics (Griffiths and Godfray 1988; Field and Calbert 1998). The females compete for uninterrupted ownership of the patch so that they can achieve parasitism and may continue to defend the patch for some time after parasitism is completed (Hughes et al. 1994; Field and Calbert 1999; Perez-Lachaud et al. 2002). Such aggression may prevent or reduce superparasitism (when a host is parasitised by more than one female) and protects the reproductive investment by the female owner in that particular patch (Field et al. 1997; Goubault et al. 2005). Aggressive defence of host patches may also protect against interspecific competition (Hardy and Blackburn 1991; Batchelor et al. 2005) and hyperparasitism (Murray et al. 2009).

When a female parasitoid first encounters a patch, she needs to assess host quality and decide whether to invest in those hosts or to continue searching for another patch. A substantial literature exists on the decisionmaking process in this scenario (Field et al. 1998; Goubault et al. 2004; van Alphen and Bernstein 2008), which applies to all parasitoids whether or not they defend patches against competitors. For species that do defend patches, if another female encounters the same patch after the first female (the owner) has begun parasitism, the owner faces a new decision. Should she: (1) share the patch while she continues parasitism; (2) leave the patch to search for another; or (3) engage in aggressive defence of the patch to retain ownership? The newly arrived female (the intruder) also faces a decision. Should she (1) start parasitism and share the patch; (2) leave the patch to search for another or (3) attack and drive away the owner to take possession of the patch? The ability to defend the patch from a competitor adds a layer of complexity to the decision-making process for the two females when they trade-off between current and future reproductive investment (Hughes et al. 1994; Field and Calbert 1998).

Time spent by a female parasitoid in aggressive behaviour at host patches reduces the time available for parasitism during that female's lifespan. If that time spent on aggression leads to a reduction in the number of hosts parasitised throughout life, the female has lost fitness. A potential consequence of a reduction in parasitism by females that engage in aggressive host defence is an associated reduction in overall parasitism of the host population. On the other hand, time spent in aggression may increase a female's fitness (and maintain or even increase suppression of host populations) if intraspecific aggression enables the female to gain access to more hosts or prevents superparasitism. Decisions that maximise parasitoid fitness are expected to increase suppression of host populations (Mills and Wajnberg 2008), but does this prediction hold when females engage in active defence of hosts?

The solitary egg parasitoid Enoggera nassaui (Girault) (Hymenoptera: Pteromalidae) was introduced to New Zealand as a biological control agent of the eucalyptus tortoise beetle Paropsis charybdis Stål (Coleoptera: Chrysomelidae) (Kay 1990). The host of E. nassaui undergoes two generations during the austral spring and summer followed by a winter diapause (Kay 1990). Parasitoid populations build up from very low levels in early spring to more than $80 \%$ parasitism by late summer (population dynamics of this host-parasitoid system are described in more detail by Mansfield et al. 2011). When E. nassaui was introduced, neither competing primary parasitoids nor hyperparasitoids were present in New Zealand. The accidental arrival in 2002 of a new competitor, the primary parasitoid Neopolycystus insectifurax Girault (Hymenoptera: Pteromalidae), and a hyperparasitoid Baeoanusia albifunicle Girault (Hymenoptera: Encyrtidae) that attacks $E$. nassaui but not $N$. insectifurax, changed the dynamics of biological control for $P$. charybdis (Murphy 2002; Berry 2003). To estimate the impact of these accidental introductions, new research was conducted on the biology of $P$. charybdis and its associated parasitoids (Jones and Withers 2003; Jones et al. 2005; Murray et al. 2008; Mansfield et al. 2011). The host range of $E$. nassaui has also been investigated post-release (Murray et al. 2010), but its parasitismrelated behaviour has not yet been described in depth.

It was during these more recent investigations that female $E$. nassaui was found to engage in active defence of host resources, something that was not recognised at the time of introduction into New Zealand. Female E. nassaui may fight if they encounter one another while engaged in parasitism, similar to Pachycrepoideus vindemiae Rondani (Hymenoptera: Pteromalidae) (Goubault et al. 2007), but unlike some parasitoids (Hardy and Blackburn 1991; Field et al. 1998), there is no evidence that females remain on egg batches to guard the developing offspring after parasitism is finished (Mansfield, personal observation). Enoggera nassaui does engage in aggression with other parasitoid species (Murray et al. 2009; Murray 2010); however, this study will focus on aggression between conspecifics.

It is useful to have a baseline description of $E$. nassaui behaviour in the absence of competitors and in response to conspecific competitors because this provides a point of reference for studies of interspecific interactions. Furthermore, the effect of female-female aggression among parasitoids on parasitism of $P$. charybdis or other paropsines has not been considered before. So far, two species of Enoggera, including E. nassaui, have been exported from Australia as biological control agents for several paropsine beetles on eucalypts in New Zealand, South Africa and the USA (Kay 1990; Tribe and Cillie 2000; 
Paine and Millar 2002). In 2007, Paropsisterna selmani Reid \& de Little was found in Ireland and is the first paropsine to reach Europe (Reid and de Little 2013). If female-female aggression is common across Enoggera spp., there may be unexpected consequences for ongoing and future biological control programmes. Aggression between conspecifics increased the time needed to complete oviposition in another parasitoid, Dinarmus basalis Rondani (Hymenoptera: Pteromalidae), and it was hypothesised that contests between females would enhance biological control by encouraging parasitoid dispersal (Mohamad et al. 2013). This parasitoid attacks a stored product pest where hosts will be aggregated within storage facilities, which is very different from the situation of $E$. nassaui females searching for a forestry pest within large-scale plantations. In a forestry environment, parasitoid efficiency may decrease if contests between $E$. nassaui increase the time taken to complete oviposition.

This study first describes the behaviour of $E$. nassaui in the absence of conspecific competitors (solo females) to provide baseline information. Next, aggressive interactions between pairs of female $E$. nassaui are described because these interactions had never been observed before and the effect of such behaviour on parasitism rates is unknown. Finally, the parasitism behaviour of solo females is compared with the parasitism behaviour of competing females (owners and intruders) to test the following hypotheses: (1) the order of arrival at a patch (owner versus intruder) will affect the time spent by an individual on parasitism-related behaviour and/or parasitism success; (2) the presence of a competitor will reduce the amount of time spent on parasitism-related behaviour for competing females compared with solo females; and (3) overall parasitism of the host population will be lower when two females are in competition compared with a solo female.

\section{Methods}

All cultures were maintained and observations performed at $22 \pm 2{ }^{\circ} \mathrm{C}, 65-70 \% \mathrm{RH}$ and 14:10 L:D.

\section{Insect cultures}

Rearing methods for $P$. charybdis and E. nassaui followed those described in Mansfield et al. (2011). Briefly, the cultures were started by collecting adult beetles and parasitised egg batches from several infested eucalypt plantations in the central North Island of New Zealand (38 $14^{\prime} 23^{\prime \prime}$ S $176^{\circ} 13^{\prime} 22^{\prime \prime}$ E). Beetles were fed Eucalyptus nitens (H. Deane and Maiden) Maiden foliage in Perspex cages $(1.0 \mathrm{~m}$ tall $\times 0.7 \mathrm{~m} \times 0.7 \mathrm{~m})$, and eggs laid on the leaf tips were collected regularly for maintenance of the parasitoid stock culture and for experimental observations (described below). Host eggs remained attached to leaf tips in naturally laid clusters (batches) when presented to parasitoids in the stock culture and in observations. Host eggs were stored at $4{ }^{\circ} \mathrm{C}$ until required; eggs used for observations had been stored for $<2$ days. Cool storage of host eggs slows down embryo development in $P$. charybdis (eggs hatch after 4-7 days at ambient temperature, Murphy 1998) and maintains host quality for parasitism (Murray 2010).

In the parasitoid stock culture, parasitised egg batches were kept in Petri dishes (90-mm diameter) and the newly emerged parasitoids were left in these Petri dishes so that mating could take place before individuals were used in observations. This was the simplest way to achieve mating because male and female E. nassaui cannot be distinguished from external morphology (Naumann 1991), so male-female pairs cannot be selected visually for mating purposes. As a result, females had contact with conspecific parasitoids that emerged at the same time, similar to what they would experience under natural conditions. Adult parasitoids in the stock culture were given pure honey as a carbohydrate source.

\section{Behavioural observations}

Either one (solo) or two (paired) parasitoids were placed into a Petri dish (90-mm diameter), and paired parasitoids were released simultaneously. There was no exit from the Petri dish, so the parasitoids remained in the dish for the entire observation. Enoggera nassaui is approximately 3$\mathrm{mm}$ long and a $P$. charybdis egg is approximately $4 \mathrm{~mm} \times$ $1 \mathrm{~mm}$, so there was sufficient space in the dish for the parasitoids to avoid contact with the egg batch if they chose to, even though parasitoids could not exit from the dish. This design was used to encourage direct competition between parasitoids and maximise the opportunity to observe aggressive interactions. Undiluted honey was given on a piece of paper towel (approximately $10 \times 10 \mathrm{~mm}$ ) and was available throughout the observation. Before the hosts were presented, the wasps were allowed to feed ad libitum for at least $15 \mathrm{~min}$ to ensure that the parasitoids were not limited by food availability (Mansfield et al. 2011).

As noted above, males and females cannot be distinguished from external morphology alone, so females were identified by female-specific behavioural responses to the host eggs (Table 1 ) in the first $10 \mathrm{~min}$ of an observation. If a parasitoid did not show a female-specific response to the host eggs within $10 \mathrm{~min}$, the parasitoid and observation were discarded. For paired parasitoids used to observe intraspecific competition, only those observations were kept where both wasps demonstrated a female-specific response to host eggs and/or engaged in physical combat over possession of the egg batch during the first $10 \mathrm{~min}$ of the observation. In practice, female $E$. nassaui responded rapidly $(<5 \mathrm{~min})$ to the presence of host eggs whether alone or in pairs (Mansfield, personal observation) although there was still a risk that some females were 
Table 1 Definitions and categories of behaviour displayed by female E. nassaui with or without a conspecific competitor present

\begin{tabular}{|c|c|c|}
\hline Category & Behaviour & Definition \\
\hline \multirow[t]{4}{*}{ Off host } & Rest & Remains still on surface of leaf or Petri dish \\
\hline & Groom & $\begin{array}{l}\text { Moves legs over antennae and/or wings in a } \\
\text { combing action }\end{array}$ \\
\hline & Walk & Walks over surface of leaf or Petri dish \\
\hline & Feed & Feeds from honey supply on paper towel \\
\hline \multirow[t]{2}{*}{ On host } & Rest & Remains still on surface of egg batch \\
\hline & Groom & $\begin{array}{l}\text { Moves legs over antennae and/or wings in a } \\
\text { combing action while standing on egg batch }\end{array}$ \\
\hline \multirow[t]{4}{*}{ Parasitism } & Drum & $\begin{array}{l}\text { Walks over surface of egg batch while tapping } \\
\text { antennae up and down on egg surface }\end{array}$ \\
\hline & Probe & $\begin{array}{l}\text { Ovipositor valves partially inserted and drilling } \\
\text { through egg shell, abdomen oriented at right } \\
\text { angles to egg surface }\end{array}$ \\
\hline & Host feed & $\begin{array}{l}\text { Feeds from hole in egg surface created by } \\
\text { probing }\end{array}$ \\
\hline & Oviposit & $\begin{array}{l}\text { Ovipositor fully inserted into egg, abdomen } \\
\text { parallel to egg surface }\end{array}$ \\
\hline \multirow[t]{5}{*}{ Defence } & Flap & Faces towards opponent and flaps wings \\
\hline & Run & Runs towards opponent \\
\hline & Flee & Runs away from opponent \\
\hline & Jostle & $\begin{array}{l}\text { Opponents lock the 'corners' of their thorax } \\
\text { together and push each other back and forth } \\
\text { across the egg batch }\end{array}$ \\
\hline & Grapple & $\begin{array}{l}\text { Opponents lock mandibles and push each other } \\
\text { back and forth across the egg batch }\end{array}$ \\
\hline
\end{tabular}

Parasitism and defence behaviour always took place on the host egg batch. Defence behaviour occurred only when a competitor was present

discarded during this screening process. No parasitoid was used more than once, and a new Petri dish was used for each observation.

Female parasitoids used in the solo observations were $3.3 \pm 0.5$ days old (mean $\pm \mathrm{se}$ ) and in the paired observations were $3.1 \pm 0.3$ days old. Females were selected at random from the stock culture except that paired females used to observe intraspecific competition were selected to be the same age, i.e. both females in a pair had emerged on the same date in the stock culture. Three-day-old female E. nassaui have reached their maximum egg complement so are not limited by egg maturation (Murray 2010). Females had no prior oviposition experience but did have contact with host remains (the egg batch from which they emerged) prior to use.

A batch of host eggs was placed gently into the Petri dish at the start of an observation. Batch size for the solo and paired observations was $6.5 \pm 0.2$ eggs (mean \pm se), which is about half the expected egg complement for a 2-day-old female E. nassaui (Mansfield et al. 2011). Egg batches were selected that were laid in a single layer across one side of the leaf surface to ensure that the entire batch fitted comfortably within the field of view of a stereomicroscope at $\times 10$ magnification and that all individual eggs were visible to the observer while being readily accessible to the parasitoid(s). This ensured that parasitoid behaviour when in contact with the host eggs could be observed continuously without movement of the Petri dish. The number of host eggs presented and number of eggs that received oviposition were recorded for each observation. Parasitism was then confirmed by incubating egg batches until colour changes indicated parasitoid development (Kay 1990).

Observations continued for $30 \mathrm{~min}$ or until all eggs in the batch had been parasitised, whichever came first. Under laboratory conditions when female $E$. nassaui are left with small numbers of hosts for extended periods, they may probe repeatedly eggs they have already parasitised (without further oviposition) causing rapid desiccation and collapse of the affected eggs (Murphy 1998; Murray et al. 2010). The censoring of observations after 30 min reduced the risk of this behaviour, which prevents accurate assessment of parasitism success. The risk of self-superparasitism (a female ovipositing into the same host more than once) was reduced by stopping observations when all eggs in the batch had been parasitised; however, superparasitism between competing (paired) females could not be avoided entirely.

Behaviours for females in the absence of competitors (solo females) were defined from a pilot study (Table 1). For the observation of intraspecific competition, the behaviours used for solo females were augmented with additional defence behaviours from a second pilot study (Table 1). When two females were observed simultaneously, the first female to make contact with the egg batch was designated the 'owner' and the second female was designated the 'intruder'.

Observations of solo females were collected from November to December 2004, and paired females were observed in January 2005 by a single observer. The process used to identify females meant that it could take several attempts before a female was identified from the stock culture and an observation completed. Only one or two $30 \mathrm{~min}$ observations were completed sequentially during the morning of any 1 day to minimise observer fatigue. All observational data were recorded in real time by using the Observer 5.0 (Noldus Information Technology, http://www.noldus.com/) on a handheld device. This is event logging software designed specifically for direct recording of behaviour. The software can calculate the duration and frequency of different behaviours for an individual observation or across groups of observations as required. It is also possible to collate behavioural sequence data, e.g. determine how often drumming was followed by probing. To summarise behavioural sequences, the probability 
of a particular transition from one behaviour to another behaviour for either all solo or all paired observations was calculated as follows: the frequency that behaviour 1 was followed by behaviour 2 divided by the total frequency of behaviour 1. For example, out of 71 occasions when solo females were resting on the host egg batch, there were 60 occasions when next behaviour was drumming, so the probability that 'rest on host' is followed by 'drum' is $60 / 71=0.85$. The observational data can also be exported from the Observer into a spreadsheet to conduct additional statistical analyses not available within the programme itself.

\section{Statistical analysis}

Differences in percentage parasitism between solo and paired females were analysed with a non-parametric Mann-Whitney rank sum test because the data failed to meet the assumptions for a parametric $t$ test. Survival analysis followed by log-rank tests was used to compare time spent on parasitism-related behaviour between (1) solo and owner females and (2) solo and intruder females, following the approach of Mohamad et al. (2013). Survival analysis was chosen to analyse the time data (behaviour durations related to oviposition events) because this type of analysis can handle unequal sample sizes, censored observations and data that are not normally distributed (Manly 1992). All statistical analyses were conducted using Sigmaplot 12.5 (Systat Software Inc., https://systatsoftware.com/products/sigmaplot/).

\section{Results}

\section{Solo females}

In the absence of conspecific females, $E$. nassaui showed a consistent pattern of behaviour, spending in total $80 \%$ of their time in contact with the egg batch $(22.5 \pm 1.4 \mathrm{~min}$, mean \pm se) including $66 \%$ on parasitism-related behaviour (Fig. 1, $n=20$ observations lasting $27.9 \pm 1.0 \mathrm{~min}$ with 14 observations censored at $30 \mathrm{~min}$ ). Solo females walked and drummed over the egg batch when it was first encountered before selecting an egg to commence parasitism (drum, probe, oviposit; Fig. 2). Host feeding was observed only on the first egg probed. Solo females subsequently probed and then oviposited into the egg on which they had host fed for all observations except one. After attack of the first egg, solo females continued parasitism of the remaining eggs (without further host feeding) sometimes separated by short periods of resting, grooming or walking about the egg batch. A few females began drumming on a previously parasitised host and then suddenly halted when they reached the point where they had oviposited before and immediately walked away to another host. This suggested females had some ability to recognise hosts they had parasitised recently even though no specific marking behaviour was observed after oviposition.

Solo females parasitised 92 out of 134 eggs (69\%) in total (4.7 \pm 0.4 eggs parasitised per female). Solo females spent $11.3 \pm 0.6 \mathrm{~min}$ per egg on the four parasitismrelated behaviours: drum, probe, hostfeed and oviposit. The time required to complete parasitism of one egg

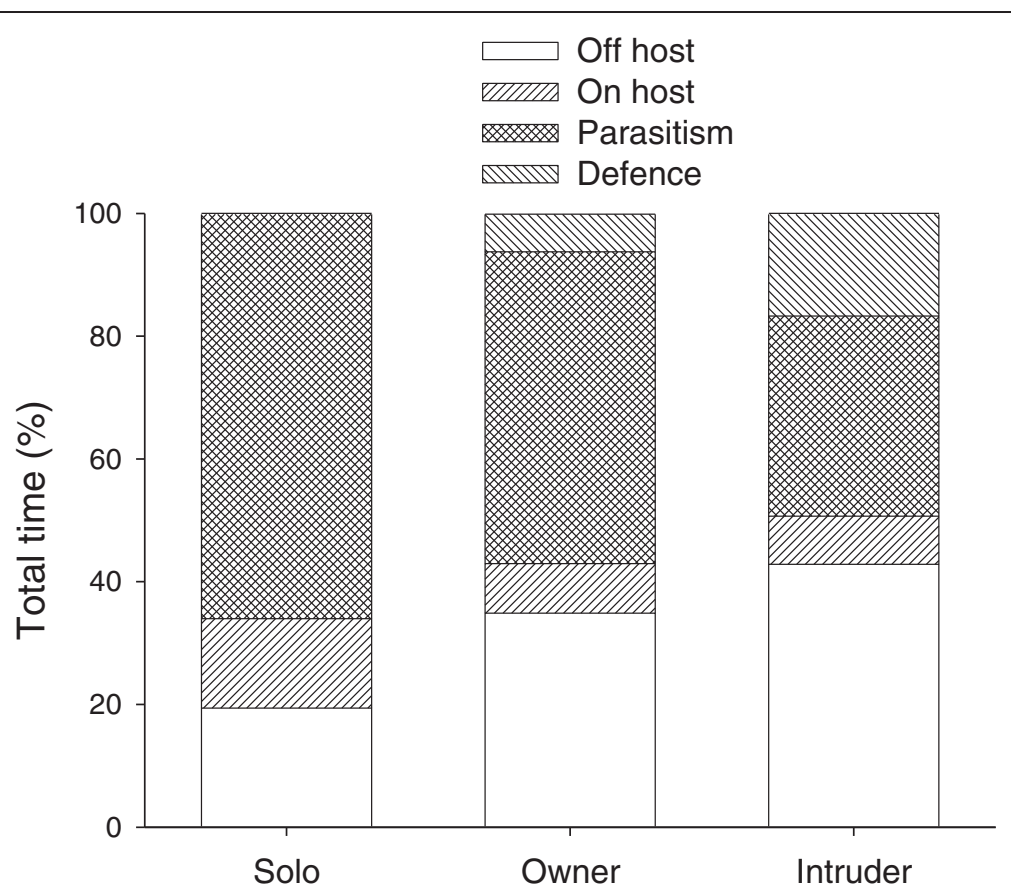

Fig. 1 Total time (\%) spent on different categories of behaviour for all observations by solo, owner and intruder E. nassaui, $n=20$ solo females and 15 owner-intruder pairs 


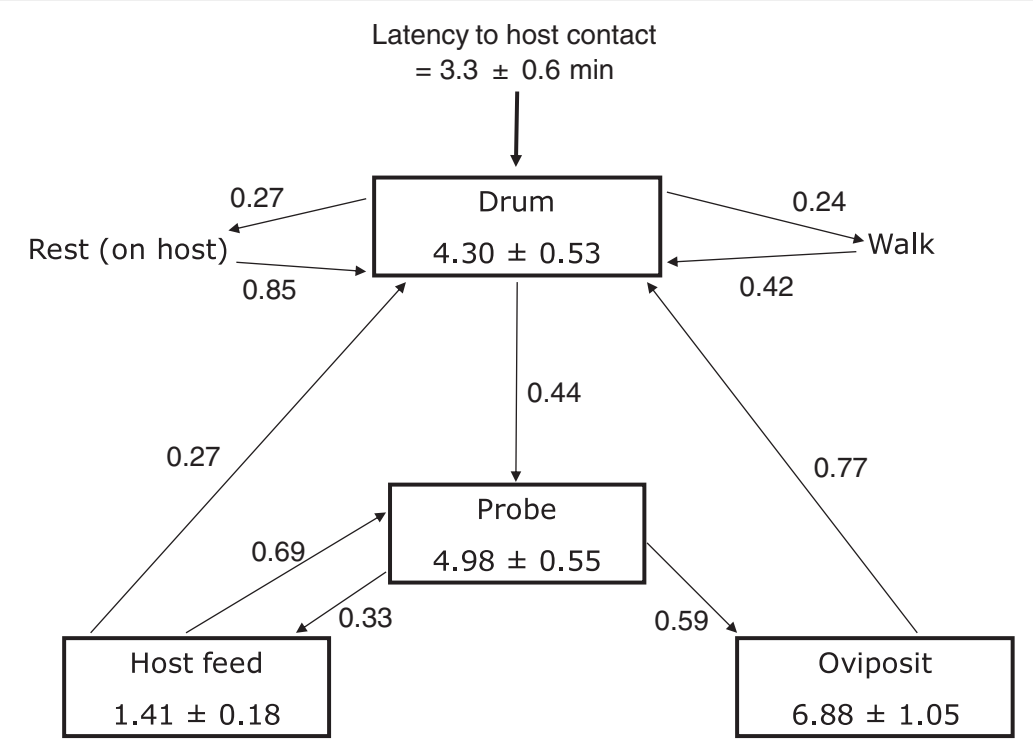

Fig. 2 General sequence of parasitism-related behaviour shown by $E$. nassaui in the absence of conspecific competitors ( $n=20$ solo females). Mean ( \pm se) total duration ( $\mathrm{min}$ ) for each behaviour is shown within boxes. Arrows indicate the direction of behavioural transitions with the probability of transition from one behaviour to the next shown beside each arrow

varied between females with some spending extended periods on a single egg and others parasitising multiple eggs in rapid succession (range $=26.8 \mathrm{~min}$ per egg). Every individual parasitised at least one egg in the batch, and six females parasitised the entire egg batch in less than $30 \mathrm{~min}$. Four solo females self-superparasitised only five eggs.

\section{Paired females}

In the presence of conspecific females, owners spent $66 \%$ of their time in contact with the egg batch including $51 \%$ on parasitism-related behaviour and $6 \%$ on defencerelated behaviour (Fig. 1, $n=15$ observations lasting 26.1 $\pm 1.5 \mathrm{~min}$ with eight observations censored at $30 \mathrm{~min}$ ). Owners were away from the egg batch for the remaining time. Intruders spent $58 \%$ of their time in contact with the egg batch including $32 \%$ on parasitism-related behaviour and $17 \%$ on defence-related behaviour (Fig. 1). Owners spent $10.0 \pm 0.8 \mathrm{~min}$ per egg on parasitism-related behaviour (range $=25.4 \mathrm{~min}$ per egg), and intruders spent $7.1 \pm 0.8 \mathrm{~min}$ per egg on parasitism-related behaviour (range $=17.1 \mathrm{~min}$ per egg). Owner and intruder females followed the sequence of parasitism-related behaviours displayed by solo females (drum, probe and oviposit with host feeding on the first egg attacked) when not interrupted by the actions of their competitor.

Paired females parasitised 65 out of 99 eggs (66 \%) in total ( $4.3 \pm 0.7$ eggs parasitised per pair). All but one of the egg batches presented had at least one egg parasitised with six egg batches parasitised completely. In one observation, both females made contact with the egg batch including drumming and probing, yet neither female oviposited into the egg batch nor they did engage in aggression (Table 2). Superparasitism by competing females occurred during 8 of the 15 observations with 13 host eggs receiving more than one oviposition. Both owners and intruders were seen to engage in this behaviour, and it was not possible to determine which of the two competing females' offspring survived following superparasitism. This made it impossible to calculate the exact number of eggs parasitised successfully by owners and intruders for all observations.

Owner status gave no apparent advantage in terms of exclusive oviposition because in 10 of the 15 observations, both owners and intruders oviposited into at least one egg within a batch (Table 2). Owners did not always respond to intruders immediately and frequently continued with parasitism-related behaviour for a time after the intruder had made her first contact with the egg batch. Intruders usually began parasitism-related behaviour when they first made contact with the egg batch, only engaging in aggression later (if at all). The level of aggression varied noticeably between pairs of females, with no interactions in six observations and five or more interactions in four observations ( $n=50$ interactions in total lasting $1.4 \pm 0.3 \mathrm{~min}$,

Table 2 Oviposition and aggression by female E. nassaui when a conspecific competitor is present ( $n=15$ owner-intruder pairs)

\begin{tabular}{lll}
\hline Oviposition & With aggression & Without aggression \\
\hline Both females & 7 & 3 \\
Owner only & 2 & 1 \\
Intruder only & 0 & 1 \\
Neither female & 0 & 1 \\
\hline
\end{tabular}


where an interaction represented one or more consecutive aggressive behaviours separated by periods of nonaggressive behaviour). Interactions often began with flap, run or jostle, which were the three most common aggressive behaviours, and often ended with jostle or grapple (Table 1, Fig. 3). Contests between females left no apparent injuries.

After an aggressive interaction finished, the owner of the batch usually remained with the egg batch and resumed parasitism-related behaviour ( $n=41$ interactions). Intruders did not show a consistent pattern of behaviour following aggressive interactions with owners. Some remained on the egg batch after the interaction was over ( $n=35$ interactions) whereas others left the egg batch after an interaction finished ( $n=14$ interactions) but returned later to engage in either further aggression or parasitism-related behaviour. Overall, possession of the egg batches could not be assigned clearly to either owner or intruder during most of these observations.

\section{Comparison of solo and paired females}

The percentage of eggs parasitised within an egg batch was similar when exposed to either one or two females (Mann-Whitney $U=150.50, \quad P=0.97$ ). Solo females spent more time on parasitism-related behaviour than intruders (log-rank test $=13.97, d f=1, P<0.001$ ) but spent similar amounts of time on parasitism as owners (log-rank test $=0.89, d f=1, P=0.34)$. Further examination showed that solo females spent more time per egg than intruders on drumming (log-rank test $=28.07$, $d f=1, P<0.001$, Table 3 ), probing (log-rank test $=4.80$, $d f=1, P=0.03$ ) and ovipositing (log-rank test $=9.33$, $d f=1, P=0.002)$ but similar amounts of time on host feeding (log-rank test $=0.10, d f=1, P=0.78)$.

\section{Discussion}

Female $E$. nassaui showed a highly consistent sequence of behaviour as they parasitised host eggs in the absence of conspecific competitors. The parasitism sequence for $E$. nassaui was similar to the behaviour of
Pachycrepoideus vindemiae (Goubault et al. 2007) and D. basalis (Mohamad et al. 2013), except for the frequency of host feeding. For the pupal parasitoid $P$. vindemiae, host feeding takes place after several hosts have been attacked, increasing the number of eggs a female can mature and/or providing energy to search for hosts (Phillips 1993; Goubault et al. 2007). Host feeding by $E$. nassaui (solo or paired) generally was confined to the first egg attacked within a batch. Usually, E. nassaui parasitised the host egg after host feeding, which suggests that the process does not damage the host such that it becomes unsuitable for larval development. Access to hosts is not essential for egg maturation in $E$. nassaui (Mansfield et al. 2011) and hosts occur naturally in batches, so the primary purpose of host feeding may be assessment of batch (host) quality with nutritional gain to the parasitoid as a secondary outcome.

Owners and intruders showed a similar consistent sequence of behaviour in relation to parasitism; however, aggression between females interrupted this behavioural sequence. Again, this is similar to Pachycrepoideus vindemiae (Goubault et al. 2007) and D. basalis (Mohamad et al. 2013). Females (whether owners or intruders) that were interrupted during the parasitism sequence before completing oviposition would usually revert back to drumming (the beginning of the parasitism sequence) when they resumed parasitism after aggressive interactions finished. This act of reverting back to previous stages of the parasitism sequence after interference from a conspecific competitor was also seen in Venturia canescens (Gravenhorst) (Hughes et al. 1994). The proportion of time allocated to defence is not reported for many parasitoids, but mymarid egg parasitoids (Gonatocerus spp.) spent no more than $2 \%$ of their time on aggressive defence of egg batches (Irvin and Hoddle 2005). In comparison, $E$. nassaui spent a relatively high proportion of time on aggressive interactions, particularly as intruders (17\%).

Aggression from E. nassaui owners did not wholly prevent parasitism by intruders, so arriving first did not guarantee exclusive use of a patch. Both owners and intruders

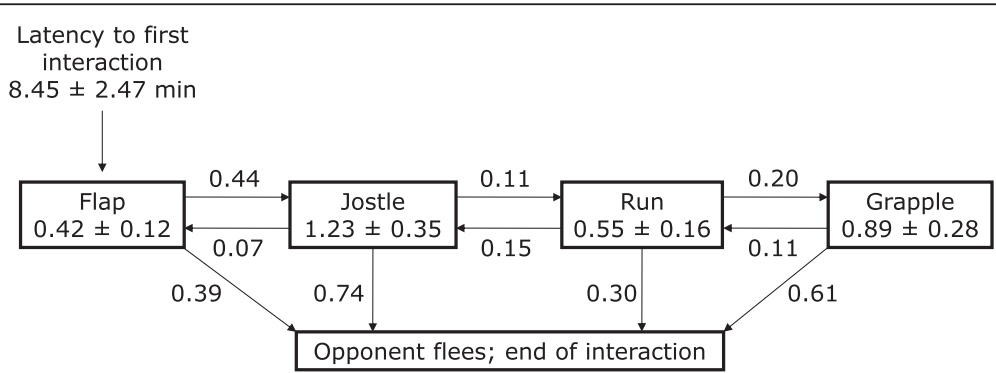

Fig. 3 General sequence of defence-related behaviour shown by E. nassaui in the presence of conspecific competitors ( $n=15$ owner-intruder pairs). Mean ( \pm se) total duration (min) for each behaviour is shown within boxes. Arrows indicate the direction of behavioural transitions with the probability of transition from one behaviour to the next shown beside each arrow 
Table 3 Time (min) spent per host egg (mean \pm se) by solo and intruder $E$. nassaui on parasitism-related behaviours ( $n=20$ solo and 11 intruder females)

\begin{tabular}{lllll}
\hline Female & Drum & Probe & Host feed & Oviposit \\
\hline Solo & $2.78 \pm 0.18$ & $3.55 \pm 0.20$ & $1.56 \pm 0.07$ & $3.37 \pm 0.32$ \\
Intruder & $1.28 \pm 0.16^{\mathrm{a}}$ & $2.72 \pm 0.31^{\mathrm{a}}$ & $1.77 \pm 0.42$ & $1.95 \pm 0.28^{\mathrm{a}}$ \\
\hline
\end{tabular}

${ }^{\mathrm{a}}$ Differs from solo females, $P<0.05$

often shared their egg batches and engaged in parasitising different eggs simultaneously. Paired females had similar prior experience of host remains, mating and contact with conspecifics in the stock culture. The key difference between owners and intruders was that owners were first to contact the egg batch with intruders second but both individuals needed to contact the egg batch within $10 \mathrm{~min}$ for the observation to continue (the possible effects of this method are discussed later). Under these conditions, potential asymmetries between competing $E$. nassaui were minimised. This may explain why it was rare for host ownership to be resolved clearly even after frequent contests. A longer period between arrival of owner and intruder may be needed to confer a consistent advantage to E. nassaui owners (Field and Calbert 1999; Mohamad et al. 2013).

Relative size may be another significant deciding factor in contests between female E. nassaui as it is in some other parasitoids (Petersen and Hardy 1996; Humphries et al. 2006). Female size does vary in E. nassaui when reared from different host species (Murray 2010), but size variation was minimal in this study because all parasitoids were reared from $P$. charybdis, the most common New Zealand host for E. nassaui. Within the native range of this parasitoid in Australia, there are many more host species present (Selman 1985), so encounters between females of different sizes would be expected to occur.

While it was rare for either owner or intruder to establish exclusive possession, aggressive interactions between E. nassaui did not escalate to the point of causing injury even when there were multiple interactions during a single observation and despite the fact that females could not exit the Petri dish. This differs from intraspecific contests between female $N$. insectifurax, another pteromalid egg parasitoid of $P$. charybdis, where injuries occur frequently (Mansfield, personal observation). Other aggressive parasitoids fight (sometimes to the death) until exclusive possession of the host is obtained and/or until parasitised hosts have reached a particular stage of development (e.g. Field et al. 1998; Batchelor et al. 2005). Further work is needed to investigate the factors that stimulate aggressive behaviour and determine contest resolution in E. nassaui, preferably with larger sample sizes than were possible in this study.

One essential step for such work will be development of isofemale lines for this species to control for kinship relationships between female parasitoids. Lize et al. (2012) demonstrated that kin recognition influences aggression between conspecific females in Goniozus legneri Gordh (Hymenoptera: Bethylidae). No attempt was made to control for kinship relationships between paired females in this study due to constraints on the availability of hosts combined with the difficulty of differentiating male and female E. nassaui.

A second step for future work will be to develop behavioural screening methods to identify female $E$. nassaui consistently before using individuals in observations. When conspecifics are present, some parasitoids may choose to wait for the owner to leave before exploiting the host (Goubault et al. 2005; Mohamad et al. 2012). Inclusion of the screening process to identify females within each observation meant this possibility was not controlled effectively and led to smaller sample sizes than were desirable.

Intruders that wait for owners to leave inevitably will need to make decisions about superparasitism, depending on batch size and the number of eggs already parasitised by the owner (Mohamad et al. 2012; Benkhellat et al. 2015). Paired E. nassaui did superparasitise occasionally, but superparasitism was not a focus of this study because the outcome of intraspecific competition among E. nassaui larvae is not known.

Solo females and owners took a similar amount of time to complete oviposition whereas intruders completed oviposition faster than solo females because intruders spent less time on three essential behaviours: drum, probe and oviposit. The presence of a competitor did not change the amount of time spent on parasitism by Eupelmus vuilleti Crawford (Hymenoptera: Eupelmidae) (Mohamad et al. 2010), but intruders did spend less time on host assessment prior to oviposition than owners (Mohamad et al. 2012). Both Pachycrepoideus vindemiae and D. basalis took longer to complete their first oviposition when conspecifics were present (Goubault et al. 2007; Mohamad et al. 2013). This suggests that a behavioural response to the presence of conspecific competitors is not unique to $E$. nassaui, but the exact nature and direction (i.e. increasing or decreasing time spent on parasitism) of such changes is species-specific.

Solo E. nassaui achieved parasitism rates that were comparable with field observations in New Zealand (Murphy and Kay 2000; Jones and Withers 2003; Mansfield et al. 2011), and overall parasitism of egg batches was similar for solo and paired females. The hypothesis that decisions that maximise parasitoid fitness should increase suppression of host populations (Mills and Wajnberg 2008) was given some support because the behavioural changes observed in the presence of competitors rendered the effect of competition neutral from the host's perspective. Higher densities of $E$. nassaui (four females per egg batch) also 
did not change parasitism rates compared with one or two females under laboratory conditions (Murray 2010). A female's perception of host density under natural conditions is expected to influence the decision to leave or stay when she encounters an egg batch already in possession of another female. The primary aims of this study were to investigate the effect of aggressive interactions on parasitism and to reveal more details about female behaviour during such interactions, when females were constrained to a single egg batch. The incidence of aggression between female E. nassaui may have been higher under these conditions compared with the natural environment where females can choose to leave egg batches that are already occupied.

\section{Conclusions}

Under natural conditions, E. nassaui competes with conspecifics and other species for hosts. These observations were designed primarily to determine the impact of direct competition between female conspecifics on parasitism rates. This led to the unexpected result that overall parasitism of hosts was similar with or without intraspecific competition because the presence of a competitor stimulated $E$. nassaui intruders to complete parasitism faster. The implication is that the presence of conspecific competitors alone is enough to trigger intruders to modify the behavioural sequence required to parasitise a host. If $E$. nassaui shows this behavioural response to interference competition under natural conditions, then direct competition and perhaps even aggression between conspecific females are unlikely to harm pest suppression by these parasitoids. The constraints on sample size in this study mean that conclusions drawn here should be considered preliminary. This work does, however, present unique observations of parasitism behaviour by an important biological control agent.

\section{Competing interests}

The author declares she has no competing interests.

\section{Acknowledgements}

This research was supported by New Zealand's Foundation for Research, Science and Technology Contract CO4X0302 and a Thompson Fellowship from the University of Sydney. Thank you to Diane Jones, Dave Hayes and Pam Taylor for their technical support. Comments from Tara Murray, Toni Withers and Thomas Hoffmeister improved the earlier drafts of this manuscript.

\section{Author details \\ 'Scion (New Zealand Forest Research Institute Ltd.), Rotorua 3046, New Zealand. ${ }^{2}$ Present Address: AgResearch, Lincoln, Private Bag 4749, Christchurch 8140, New Zealand. ${ }^{3}$ Bio-Protection Research Centre, Lincoln University, PO Box 85084, Lincoln 7647, New Zealand.}

Received: 10 August 2015 Accepted: 18 February 2016

Published online: 26 February 2016

\section{References}

Batchelor, T. P., Hardy, I. C. W., Barrera, J. F., \& Perez-Lachaud, G. (2005). Insect gladiators II: competitive interactions within and between bethylid parasitoid species of the coffee berry borer, Hypothenemus hampei (Coleoptera: Scolytidae). Biological Control, 33, 194-202.
Benkhellat, O., Jaloux, B., Moali, A., Chevrier, C., \& Monge, J. P. (2015). Host discrimination and egg laying in Anisopteromalus calandrae (Hymenoptera: Pteromalidae) ectoparasitoid of Callosobruchus maculatus (Coleoptera: Chrysomelidae). Journal of Stored Products Research, 61, 48-53.

Berry, J. A. (2003). Neopolycystus insectifurax Girault (Hymenoptera: Pteromalidae) is established in New Zealand, but how did it get here? New Zealand Entomologist, 26, 113-114

Field, S. A., \& Calbert, G. (1998). Patch defence in the parasitoid wasp Trissolcus basalis: when to begin fighting? Behaviour, 135, 629-642.

Field, S. A., \& Calbert, G. (1999). Don't count your eggs before they're parasitized: contest resolution and the trade-offs during patch defense in a parasitoid wasp. Behavioral Ecology, 10, 122-127.

Field, S. A., Keller, M. A., \& Calbert, G. (1997). The pay-off from superparasitism in the egg parasitoid Trissolcus basalis, in relation to patch defence. Ecological Entomology, 22, 142-149.

Field, S. A., Calbert, G., \& Keller, M. A. (1998). Patch defence in the parasitoid wasp Trissolcus basalis (Insecta: Scelionidae): the time structure of pairwise contests, and the 'waiting game'. Ethology, 104, 821-840.

Goubault, M., Fourrier, J., Krespi, L., Poinsot, D., \& Cortesero, A. M. (2004). Selection strategies of parasitised hosts in a generalist parasitoid depend on patch quality but also on host size. Journal of Insect Behavior, 17, 99-113.

Goubault, M., Outreman, Y., Poinsot, D., \& Cortesero, A. M. (2005). Patch exploitation strategies of parasitic wasps under intraspecific competition. Behavioral Ecology, 16, 693-701.

Goubault, M., Cortesero, A. M., Poinsot, D., Wajnberg, E., \& Boivin, G. (2007). Does host value influence female aggressiveness, contest outcome and fitness gain in parasitoids? Ethology, 113, 334-343.

Griffiths, N. T., \& Godfray, H. C. J. (1988). Local mate competition, sex ratio and clutch size in bethylid wasps. Behavioural Ecology and Sociobiology, 22, 211-217.

Hardy, I. C. W., \& Blackburn, T. M. (1991). Brood guarding in a bethylid wasp. Ecological Entomology, 16, 55-62.

Hughes, J. P., Harvey, I. F., \& Hubbard, S. F. (1994). Host-searching behavior of Venturia canescens (Grav.) (Hymenoptera: Ichneumonidae): interference- - the effect of mature egg load and prior behavior. Journal of Insect Behavior, 7, 433-454.

Humphries, E. L., Hebblethwaite, A. J., Batchelor, T. P., \& Hardy, I. C. W. (2006). The importance of valuing resources: host weight and contender age as determinants of parasitoid wasp contest outcomes. Animal Behaviour, 72, 891-898.

Irvin, N. A., \& Hoddle, M. S. (2005). The competitive ability of three mymarid egg parasitoids (Gonatocerus spp.) for glassy-winged sharpshooter (Homalodisca coagulata) eggs. Biological Control, 34, 204-214.

Jones, D. C., \& Withers, T. M. (2003). The seasonal abundance of the newly established parasitoid complex of the eucalyptus tortoise beetle (Paropsis charybdis). New Zealand Plant Protection, 56, 51-55.

Jones, D. C., Withers, T. M., \& Mansfield, S. (2005). Current status of Paropsis charybdis in the central North Island. Forest Health News, 150, 2.

Kay, M. (1990). Success with biological control of the eucalyptus tortoise beetle, Paropsis charybdis. What's New in Forest Research, Issue, 184, 4.

Lize, A., Khidr, S. K., \& Hardy, I. C. W. (2012). Two components of kin recognition influence parasitoid aggression in resource competition. Animal Behaviour, 83, 793-799.

Manly, B. F. J. (1992). The design and analysis of research studies. Cambridge, UK: Cambridge University Press.

Mansfield, S., Murray, T. J., \& Withers, T. M. (2011). Will the accidental introduction of Neopolycystus insectifurax improve biological control of the eucalyptus tortoise beetle, Paropsis charybdis, in New Zealand? Biological Control, 56, 30-35.

Mills, N. J., \& Wajnberg, E. (2008). Optimal foraging behavior and efficient biological control methods. In E. Wajnberg, C. Bernstein, \& J. J. M. van Alphen (Eds.), Behavioral ecology of insect parasitoids (pp. 3-30). Malden MA: Blackwell Publishing.

Mohamad, R., Monge, J.P., \& Goubault, M. (2010). Can subjective resource value affect aggressiveness and contest outcome in parasitoid wasps? Animal Behaviour, 80, 629-636.

Mohamad, R., Monge, J.-P., \& Goubault, M. (2012). Wait or fight? Ownership asymmetry affects contest behaviors in a parasitoid wasp. Behavioural Ecology, 23, 1330-1337.

Mohamad, R., Monge, J.P., \& Goubault, M. (2013). Do resource value and ownership status affect intensity and resolution of contests in a parasitoid wasp? Entomologia Experimentalis et Applicata, 147, 99-109.

Murphy, B. D. (1998). Effectiveness of Enoggera nassaui Girault as a biological control agent of Paropsis charybdis Stal. Masters thesis. Hamilton, New Zealand: University of Waikato. 
Murphy, B. D. (2002). New insect threatens control of eucalyptus tortoise beetle. Forest Health News, 117, 2.

Murphy, B. D., \& Kay, M. K. (2000). Paropsis charybdis defoliation of Eucalyptus stands in New Zealand's central North Island. New Zealand Plant Protection, 53, 334-338.

Murray, T. J. (2010). Influence of physiological and behavioural characteristics of parasitoids on host specificity testing outcomes and the biological control of Paropsis charybdis. PhD thesis. Lincoln, New Zealand: Lincoln University.

Murray, T. J., Withers, T. M., Mansfield, S., \& Bain, J. (2008). Distribution and current status of natural enemies of Paropsis charybdis in New Zealand. New Zealand Plant Protection, 61, 185-190.

Murray, T. J., Mansfield, S., \& Withers, T. M. (2009). Comparing the behavioural strategies of two parasitoid wasps: is aggressive resource defending good for biological control? In P. G. Mason, D. R. Gillespie, \& C. Vincent (Eds.), Proceedings of the Third International Symposium on Biological Control of Arthropods (pp. 416-420). Christchurch, New Zealand: USDA Forest Service.

Murray, T. J., Withers, T. M., \& Mansfield, S. (2010). Choice versus no-choice test interpretation and the role of biology and behaviour in parasitoid host specificity tests. Biological Control, 52, 153-159.

Naumann, I. D. (1991). Revision of the Australian genus Enoggera Girault (Hymenoptera: Pteromalidae: Asaphinae). Journal of the Australian Entomological Society, 30, 1-17.

Paine, T. D., \& Millar, J. G. (2002). Insect pests of eucalypts in California: implications of managing invasive species. Bulletin of Entomological Research, 92, 147-152.

Perez-Lachaud, G., Hardy, I. C. W., \& Lachaud, J.P. (2002). Insect gladiators: competitive interactions between three species of bethylid wasps attacking the coffee berry borer, Hypothenemus hampei (Coleoptera: Scolytidae). Biological Control, 25, 231-238.

Petersen, G., \& Hardy, I. C. W. (1996). The importance of being larger: parasitoid intruder-owner contests and their implications for clutch size. Animal Behaviour, 51, 1363-1373.

Phillips, D. S. (1993). Host-feeding and egg maturation by Pachycrepoideus vindemiae. Entomologia Experimentalis et Applicata, 69, 75-82.

Reid, C. A. M., \& De Little, D. W. (2013). A new species of Paropsisterna Motschulsky, 1860, a significant pest of plantation eucalypts in Tasmania and Ireland (Coleoptera: Chrysomelidae: Chrysomelinae). Zootaxa, 3681, 395-404.

Selman, B. J. (1985). The evolutionary biology and taxonomy of the Australian eucalyptus beetles. Entomography, 3, 451-454.

Tribe, G. D., \& Cillie, J. J. (2000). Biological control of the Eucalyptus-defoliating Australian tortoise beetle Trachymela tincticollis (Blackburn) (Chrysomelidae: Chrysomelini: Paropsina) in South Africa by the egg parasitoid Enoggera reticulata Naumann (Hymenoptera: Pteromalidae: Asaphinae). African Entomology, 8, 15-22.

van Alphen, J. J. M., \& Bernstein, C. (2008). Information acquisition, information processing, and patch time allocation in insect parasitoids. In E. Wajnberg, C. Bernstein, \& J. J. M. van Alphen (Eds.), Behavioral ecology of insect parasitoids (pp. 172-192). Malden MA: Blackwell Publishing.

\section{Submit your manuscript to a SpringerOpen ${ }^{\circ}$ journal and benefit from:}

- Convenient online submission

- Rigorous peer review

- Immediate publication on acceptance

- Open access: articles freely available online

- High visibility within the field

- Retaining the copyright to your article 